\title{
Revascularização do miocárdio com a artéria radial
}

\author{
Luís Alberto DALLAN*, Sérgio Almeida de OLIVEIRA*, Ricardo C. CORSO*, Ana N. PEREIRA*, José \\ Carlos R. IGLÉSIAS*, Geraldo VERGINELLI*, Adib D. JATENE*
}

DALLAN, L. A.; OLIVEIRA, S. A.; CORSO, R. C.; PEREIRA, A. N.; IGLÉSIAS, J. C. R.; VERGINELLI, G.; JATENE, A. D. - Revascularizaçāo do miocárdio com a artéria radial. Rev. Bras. Cir. Cardiovasc., 10 (2): $77-83,1995$.

RESUMO: Os enxertos com a artéria radial foram utilizados há mais de 20 anos e praticamente abandonados após constatação de elevadas taxas de oclusão ou estenose em estudos pós-operatórios. Mais recentemente, seu emprego foi reiniciado associado ao uso dos bloqueadores dos canais de cálcio. Nos últimos 6 meses, em 30 pacientes realizamos 31 enxertos aorto-coronários coma artéria radial. Concomitantemente, a artéria torácica interna esquerda foi empregada em todos $(100 \%)$ os pacientes, a artéria torácica interna direita em $9(30 \%)$ pacientes e em $24(80 \%)$ também foram realizadas pontes de veia safena. A média de enxertos por paciente foi de 3,5 . O enxerto de artéria radial foi realizado para a artéria diagonal em $10(33,3 \%)$ pacientes, artéria circunflexa em $8(26,6 \%)$, artéria coronária direita em $8(26,6 \%)$, artéria diagonalis em 4 $(13,3 \%)$ e artéria interventricular anterior em $1(3,3 \%)$. Quatorze $(46,6 \%)$ pacientes tinham antecedentes de infarto do miocárdio e em $2(6,6 \%)$ tratava-se de reoperação. Em 3 pacientes realizou-se a endarterectomia coronária e uma dessas artérias recebeu enxerto com artéria radial. A artéria radial esquerda foi utilizada em $28(93,4 \%)$ pacientes e a direita em $2(6,6 \%)$. A artéria radial foi anastomosada à aorta ascendente como enxerto livre, diretamente com sutura contínua de Polipropileno 7-0. Completada a anastomose, a pinça da aorta era removida, para avaliação do fluxo sangūineo através da artéria radial. Todos os pacientes receberam nifedipina no intra e no pós-operatório, quando se associou o AAS. O calibre da artéria radial variou de $2,5 \mathrm{~mm}$ a $3,75 \mathrm{~mm}$ e nenhum paciente apresentou sinais de isquemia ou outras alteraçōes na mão. Não houve mortalidade nesta série, nem complicaçōes per-operatórias conseqũentes ao uso do enxerto. 0 cateterismo precoce, antes da alta hospitalar, foi realizado em $7(23,3 \%)$ pacientes e em todos o enxerto encontrava-se pérvio e sem espasmo (um deles para a artéria submetida a endarterectomia). A artéria radial parece constituir uma alternativa de grande importância na revascularização do miocárdio, especialmente após o advento dos bloqueadores dos canais de cálcio. Entretanto, é necessário maior número de estudos e o seguimento a longo prazo dos pacientes, para conclusőes definitivas.

DESCRITORES: Revascularização do miocárdio, cirurgia, artéria radial. Artéria radial, revascularizaçāo do miocárdio.

\section{INTRODUÇÃO}

O emprego da artéria radial (AR) na revascularização do miocárdio foi proposto por CARPENTIER et alii ${ }^{5}$, em 1971 . Decorridos 2 anos, os próprios autores desaconselharam o método, pois controles arteriográficos demonstraram $35 \%$ de estenoses ou oclusões nos enxertos ${ }^{4}$. Essas com- plicações decorriam principalmente de espasmo na AR desnervada.

Entretanto, passados 15 anos, constatou-se que alguns enxertos radiais, considerados ocluídos ou com graus variáveis de obstrução no pós-operatório imediato, encontravam-se pérvios e isentos de lesões ateroscleróticas ${ }^{3}$.

Trabalho realizado no Instituto do Coração do Hospital das Clínicas da Faculdade de Medicina da Universidade de São Paulo. São Paulo, SP, Brasil. Apresentado ao $22^{\circ}$ Congresso Nacional de Cirurgia Cardiaca. Brasília, DF, 30 de março a $1^{\circ}$ de abril, 1995.

* Do Instituto do Coração do Hospital das Clínicas da Faculdade de Medicina da Universidade de São Paulo.

Endereço para correspondência: Luís Alberto Dallan. Rua Inhambu, 917, cj. 191. CEP 04520-013 São Paulo, SP, Brasil. Fax: [011] 570.7802. 
DALLAN, L. A.; OlIVEIRA, S. A.; CORSO, R. C.; PEREIRA, A. N.; IGLÉSIAS, J. C. R.; VERGINELLI, G.; JATENE, A. D. Revascularizaçāo do miocárdio com a artéria radial.

Rev. Bras. Cir. Cardiovasc., 10 (2): 77-83, 1995.

Essas observações e o advento de novas drogas que atuam sobre o espasmo arterial, têm estimulado a retomada do enxerto com AR na revascularização do miocárdio.

\section{CASUÍSTICA E MÉTODOS}

De maio a novembro de 1994, 30 pacientes receberam 31 enxertos de $A R$ na revascularização do miocárdio. Simultaneamente, a artéria torácica interna esquerda (ATIE) foi empregada em todos $(100 \%)$ os pacientes. A artéria torácica interna direita (ATID) foi utilizada em $9(30 \%)$ desses pacientes e em $24(80 \%)$ pacientes também foram realizadas pontes de veia safena. A média de enxertos por paciente foi de 3,5 . O enxerto de AR foi realizado para a artéria diagonal em 10 (33,3\%) pacientes, artéria marginal esquerda em $8(26,6 \%)$, artéria coronária direita em $8(26,6 \%)$, artéria diagonalis em $4(13,3 \%)$ e artéria interventricular anterior em $1(3,3 \%)$. A faixa etária variou de 38 anos a 74 anos ( $M=56,9$ anos), com predomínio do sexo masculino em $23(76,6 \%)$ pacientes. Como principais antecedentes pré-operatórios, destacamos hipertensão arterial sistêmica $(17 / 56 \%)$, tabagismo $(12 / 40 \%)$, dislipidemia $(8 / 26,6 \%)$ e diabetes $(8 /$ $26,6 \%$ ). Quatorze $(46,6 \%)$ pacientes tinham antecedentes de infarto do miocárdio e em $2(6,6 \%)$ tratava-se de reoperação. Em 3 pacientes realizou-se a endarterectomia coronária e uma dessas artérias recebeu enxerto com AR. Em 2 pacientes associouse a aneurismectomia de ventrículo esquerdo. A AR esquerda foi utilizada em $28(93,4 \%)$ pacientes e a direita em $2(6,6 \%)$.

A manobra de Allen modificada foi realizada rotineiramente no pré-operatório, visando avaliar a circulação arterial do membro superior. Em nenhum dos pacientes suspeitou-se de malformação das artérias radial, ulnar ou do arco palmar. Demos preferência à retirada do enxerto do membro não dominante e sem cateterismo arterial prévio. A retirada do enxerto radial foi realizada simultaneamente à toracotomia.

A incisão cirúrgica foi realizada na face interna do antebraço, a partir de $4 \mathrm{~cm}$ abaixo da prega do cotovelo e terminando $3 \mathrm{~cm}$ antes da prega do pulso. Após seccionar-se pele e tecido celular subcutâneo, é exposta a AR em toda sua extensão. Em todos os casos a artéria interóssea é preservada. A AR é removida em forma de pedículo, incluindo 2 veias satélites e com a maior quantidade possível do tecido gorduroso e da tênue fascia que a recobre (superior e inferiormente). Os ramos colaterais são ligados individualmente com fios de algodão 4-0, junto ao pedículo e distalmente ao mesmo, permitindo sua secção sem traumatismos. Realizamos de 70 a 80 ligaduras em cada caso. A hemostasia do enxerto já instalado é complementada com clipes metálicos.

Após total dissecção do pedículo radial, testamos o pulso retrógrado, promovendo a oclusão temporária da artéria, próxima à sua porção umeral. Em seguida, a artéria é seccionada nessa região, o que permite avaliar seu refluxo, oriundo da artéria umeral e do arco palmar. A artéria é, então, retirada e sua luz imediatamente lavada com solução salina morna, contendo papaverina e pequena quantidade de heparina (para evitar precipitação). Essa manobra é realizada com o auxílio de um jelco número 22 , suavemente introduzido através de uma de suas extremidades (sem ligadura) e evitando-se exercer pressão lesiva ao endotélio. O enxerto é mantido imerso nessa solução até a sua utilização.

A AR foi anastomosada à aorta ascendente como enxerto livre, com sutura contínua de Polipropileno 7-0. Complementada a anastomose, a pinça da aorta era removida para avaliação do fluxo sangüíneo. Todos os pacientes receberam nifedipina no intra e no pós-operatório, quando se associou o AAS. O calibre da $A R$ variou de $2,5 \mathrm{~mm}$ a $3,75 \mathrm{~mm}$, sua extensão foi de $12 \mathrm{~cm}$ a $18 \mathrm{~cm}$ e não observamos calcificações ou outros processos degenerativos.

O estudo histológico foi realizado rotineiramente na extremidade do enxerto, junto ao punho, visando estudar a estrutura do vaso (Figuras 1, 2).

\section{RESULTADOS}

Não houve mortalidade nesta série. Em $2(6,6 \%)$ pacientes houve suspeita clínica de IAM trans-operatório devido a alterações eletrocardiográficas e/ou elevação nos níveis séricos de CKMB. Em ambos,

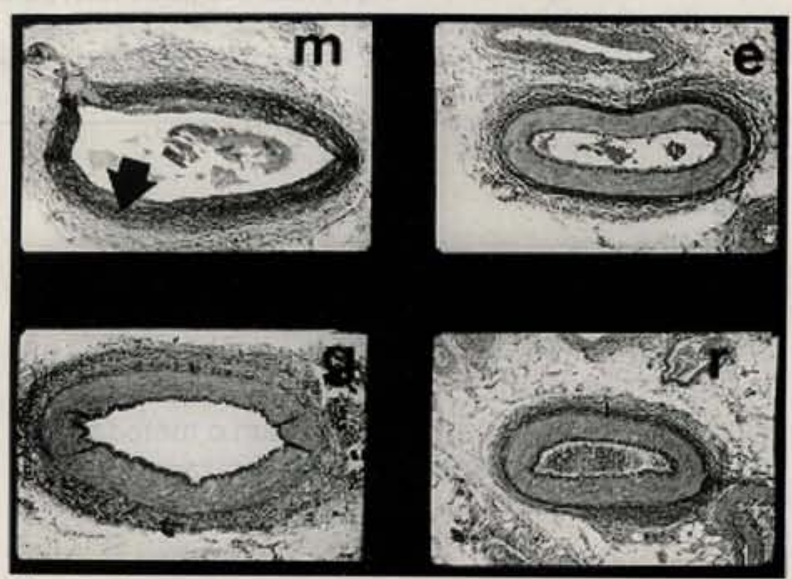

Fig. 1 - Corte transversal ( $\times 15$ ) de 4 condutos arteriais impregnados pela prata, para demonstração de fibras elásticas: artéria torácica interna $(\mathrm{m})$, art. epigástrica $(\mathrm{e})$, art. gastroepiplóica (g) e art. radial (r). Destaca-se a menor expessura da camada média da art. torácica interna $(\mathrm{m})$ sobre as demais. (seta $)$. 
DALLAN, L. A.; OliVeirA, S. A.; CORSO, R. C.; PEREIRA, A. N.; IGLÉSIAS, J. C. R.; VERGINELLI, G.; JATENE, A. D. Revascularização do miocárdio com a artéria radial.

Rev. Bras. Cir. Cardiovasc., 10 (2): 77-83, 1995.

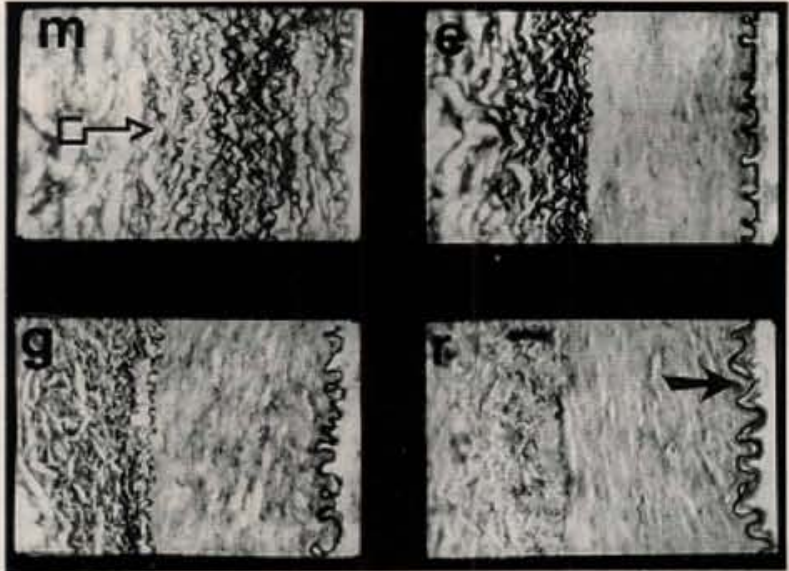

Fig. 2 - Aspecto histológico em grande aumento (X 200) das 4 artérias. Destaca-se o maior número de fibras elásticas na artéria torácica interna $(\mathrm{m})$ (seta )e áreas de descontinuidade na lâmina elástica interna da artéria radial (r) (seta ).

o estudo com pirofosfato resultou negativo. Em um deles, a área de corrente de lesão correspondia à do enxerto radial. Este paciente foi submetido a estudo cinecoronariográfico, que demonstrou estar o enxerto radial pérvio e sem espasmos, com função contrátil do ventrículo esquerdo preservada.

O cateterismo precoce (antes da alta hospitalar) foi realizado num total de $7(23,3 \%)$ pacientes e, em todos, o enxerto com a artéria radial encontrava-se pérvio e sem espasmos (um deles para a artéria submetida a endarterectomia) (Figuras 3, 4, $5,6,7)$. Num dos pacientes houve suspeita de dissecção do terço inicial da artéria radial pela ponta do cateter, que, entretanto, se estabilizou, sem a oclusão do enxerto. Nos demais pacientes, foram realizados estudos com pirofosfato e/ou tálio, sem que houvesse sinal de isquemia na área miocárdica correspondente ao enxerto radial.

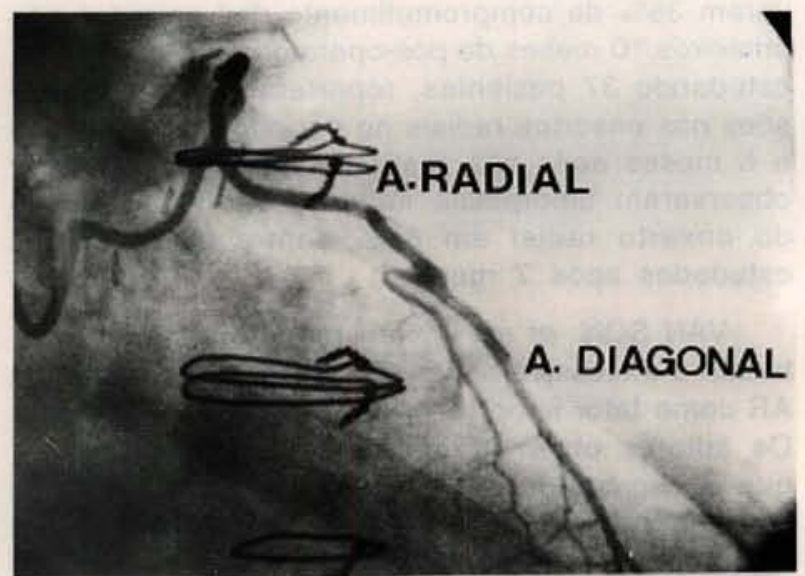

Fig. 3 - Estudo angiográfico precoce (antes da alta hospitalar) mostrando enxerto pérvio de artéria radial realizado para a artéria diagonal.

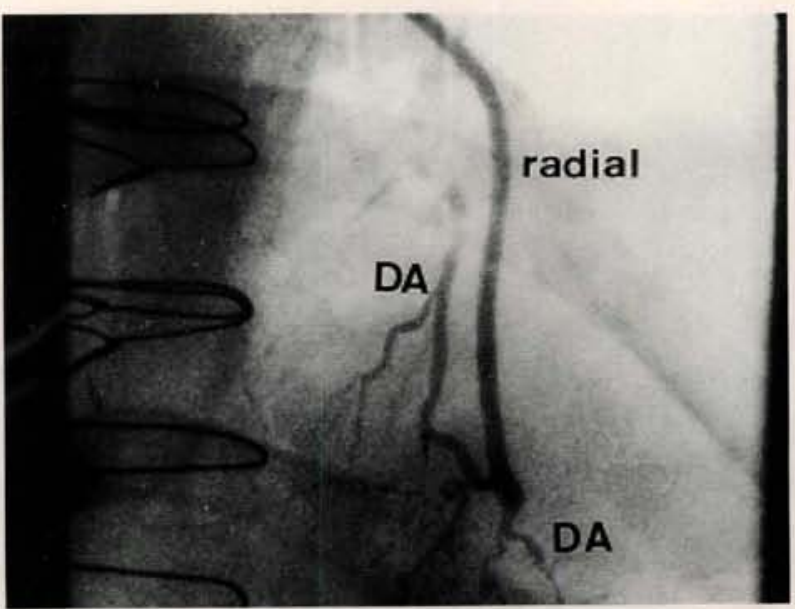

Fig. 4 - Enxerto de artéria radial para a artéria interventricular anterior (DA) (sete dias de pós-operatório).

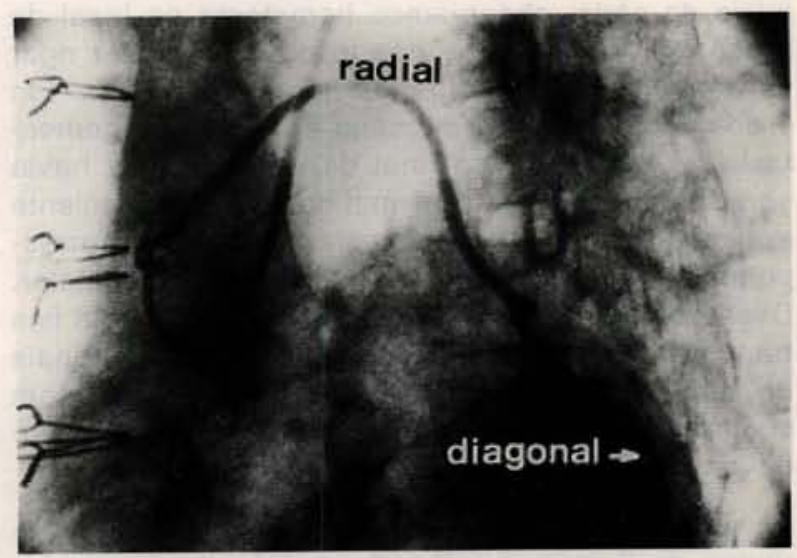

Fig. 5 - Cineangiografia: artéria patente e ausência de espasmos na artéria radial para a artéria diagonal (sexto dia de pósoperatório).

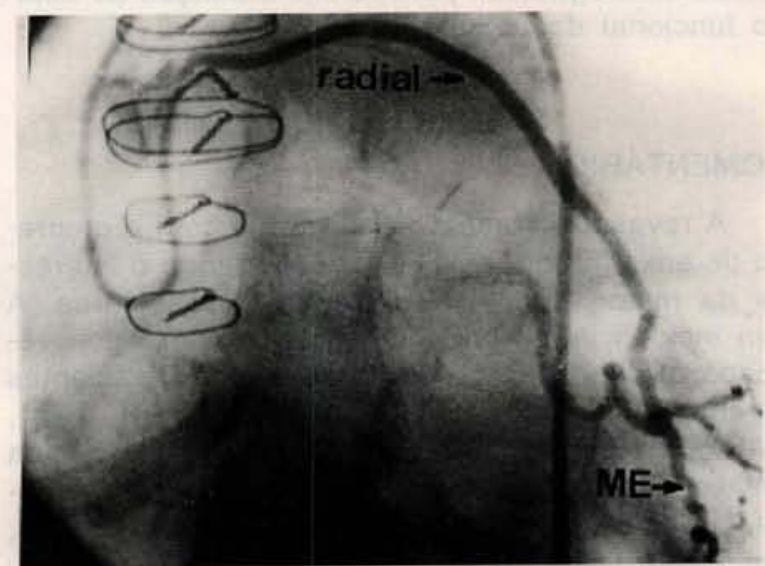

Fig. 6 - Enxerto de artéria radial para o ramo marginal esquerdo da artéria circunflexa (seta).

A alta hospitalar ocorreu em período de 7 dias a 12 dias (média=8,3 dias). Nenhum paciente apresentou infecção hospitalar ou necessidade de revisão de hemostasia torácica. Em 3 pacientes do 
DALLAN, L. A.; OlIVEIRA, S. A.; CORSO, R. C.; PEREIRA, A. N.; IGLÉSIAS, J. C. R.; VERGINELLI, G.; JATENE, A. D. Revascularização do miocárdio com a artéria radial.

Rev. Bras. Cir. Cardiovasc., 10 (2): 77-83, 1995.

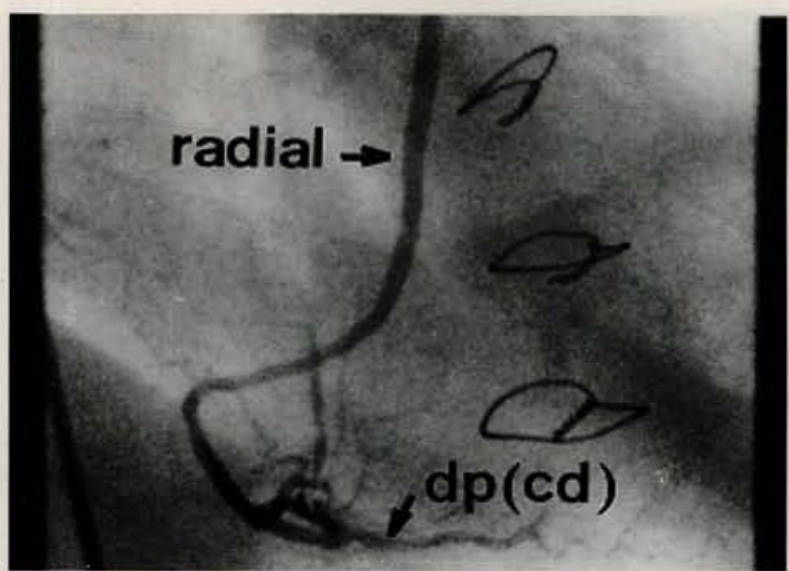

Fig. 7 - Aspecto angiográfico do enxerto de artéria radial para o ramo interventricular posterior (dp) da artéria coronária direita (seta).

início da série, observamos hematoma no local da incisão cirúrgica, o que nos levou a um maior rigor na hemostasia dos cotos dos ramos colaterais. Em 1 dos pacientes foi necessária a revisão de hemostasia no antebraço ao final da cirurgia, pois havia se soltado a ligadura proximal da AR. Esse paciente apresentou discreta parestesia no polegar correspondente, que desapareceu até a alta hospitalar. Desde então, os cotos arteriais são ligados com fios transfixantes. Nenhum paciente apresentou sinais de isquemia na mão. Não houve mortalidade, nem complicações per-operatórias conseqüentes ao uso do enxerto.

Esses pacientes vêm sendo observados por períodos de 3 meses a 9 meses. Não houve mortalidade ou recidiva de sintomas anginosos. Estão programados novos estudos rádio-isotópicos e cinecoronariográficos para futura avaliação do estado funcional do enxerto.

\section{COMENTÁRIOS}

A revascularização do miocárdio com o emprego de enxertos arteriais vem despertando o interesse da maioria dos cirurgiōes cardiovasculares. A alta incidência de problemas com os enxertos venosos a longo prazo, especialmente em pacientes jovens, tem motivado essa busca. Já em 1971, CARPENTIER et alii 5 iniciaram o uso da AR na revascularização do miocárdio. Nessa mesma década, o Dr. Domingos Junqueira de Moraes utilizou, em nosso meio, o enxerto com AR na revascularização do miocárdio*. Infelizmente, o método foi abandonado poucos anos depois, devido à constatação de elevadas taxas de oclusão ou estenose, em estudos pós-operatórios ${ }^{4}$. Carpentier sugeriu

"MORAES, Domingos Junqueira. Comunicaçāo pessoal. que o espasmo da AR estaria relacionado à falta de inervação e condenou o método até que esse problema pudesse ser solucionado.

O advento de novas drogas antiespasmódicas arteriais, especialmente os bloqueadores dos canais de cálcio, e a constatação de que vários enxertos com AR considerados ocluídos na década de 70 se mostravam com excelente perviabilidade em controles angiográficos realizados mais de 15 anos depois, novo despertaram interesse pela AR.

Resultados preliminares de estudos realizados por ACAR et alii ${ }^{3}$ colocam a AR como um enxerto complementar às artérias torácicas internas e uma alternativa a outros tipos de enxertos.

O emprego da AR apresenta algumas vantagens. Ao contrário da veia safena, sua estrutura já está adaptada ao regime de pressão arterial sistêmica; o diâmetro é superior ao dos demais enxertos arteriais e a estrutura da parede facilita tecnicamente a anastomose com a aorta e com a artéria coronária. A consistência da sua parede também facilita a moldagem intrapericárdica, mesmo quando exuberante em seu comprimento, prevenindo os acotovelamentos que fatalmente ocorreriam com a veia safena. Seu comprimento (que foi de até $18 \mathrm{~cm}$ em nossa casuística) permite atingir com folga todos os vasos da superfície cardiaca, mesmo nos grandes ventrículos. Pode-se, ainda, realizar dois enxertos com uma mesma artéria e utilizar-se da AR de ambos os braços, especialmente em pacientes com dificuldades na obtenção dos enxertos habituais, devido à safenectomia, ou subsmetidos a reoperações cardíacas.

Apesar dessas vantagens incontestáveis, devemos ter em mente a sua estrutura excessivamente "muscular", que deve ter contribuido para os insucessos descritos na literatura. CARPENTIER et alii ${ }^{5}$, em sua primeira série de 42 pacientes, observaram $35 \%$ de comprometimento dos enxertos nos primeiros 10 meses de pós-operatório. FISK et alii ${ }^{11}$, estudando 37 pacientes, reportaram $50 \%$ de oclusões nos enxertos radiais no período de 2 semanas a 6 meses após sua realização. CURTIS et alii ${ }^{9}$ observaram hiperplasia intimal levando à oclusão do enxerto radial em $65 \%$ dentre 29 pacientes estudados após 7 meses.

VAN SON et alii ${ }^{23}$, em recente editorial, destacam a excessiva espessura da camada média da AR como fator importante no insucesso do enxerto. Os autores observaram, em estudos histológicos, que a espessura da camada média da AR mede aproximadamente $500 \mu \mathrm{m}$, enquanto obtiveram valores respectivamente de $300 \mu \mathrm{m}$ para a ATI 280 $\mu \mathrm{m}$ para a artéria gastroepiplóica direita (AGED) e $240 \mu \mathrm{m}$ para a artéria epigástrica inferior $(\mathrm{AEI})^{22}$. 
DALLAN, L. A.; OliveIRA, S. A.; CORSO, R. C.; PEREIRA, A. N.; IGLÉSIAS, J. C. R.; VERGINELLI, G.; JATENE, A. D. Revascularização do miocárdio com a artéria radial. Rev. Bras. Cir. Cardiovasc., 10 (2): 77-83, 1995.

Além disso, VAN SON et alii ${ }^{23}$ observaram que a nutrição da camada média da AR é mantida preferencialmente através da difusão do sangue contido em sua luz, uma vez que não verificaram a penetração de vasa-vasorum nessa camada. Dessa maneira, os autores consideram que a espessura excesisva da camada média poderia predispô-la a graus de isquemia, especialmente em sua porção mais externa, desencadeando sua fibrose. Por outro lado, foi observado considerável número de descontinuidades na lâmina elástica da AR, com leve ou moderada hiperplasia intimal 21. Esse aspecto torna a AR mais vulnerável que a ATI, que possui múltiplas lamelas elásticas em sua camada média e menor hiperplasia intimal.

ACAR \& BRUNEVAL ${ }^{1}$, em recente casuística, não compartilham com as afirmações acima colocadas, especialmente quanto à hiperplasia intimal. Os autores consideram que esse fenômeno é observado na maioria dos enxertos, inclusive na ATI, cujos resultados favoráveis são amplamente conhecidos. A AR constitucionalmente apresenta maior hiperplasia da camada média em relação às outras artérias comumente empregadas, devido às suas densas linhas de leiomiócitos. Entretanto, não podemos esquecer que o diâmetro interno da $A R$ é muito superior ao das demais artérias. O diâmetro médio de AR estudada histologicamente por esses autores foi de $2,7 \mathrm{~mm}$ versus $1,7 \mathrm{~mm}$ para a ATI. Observaram, ainda, que a descontinuidade da lâmina elástica interna entre a camada íntima e média não é característica isolada da AR, ocorrendo, também, nas demais artérias, inclusive na ATI ${ }^{2}$. A causa potencial da falência do enxerto radial estaria relacionada à sua alta propenção ao espasmo, já demonstrado in vitro ${ }^{13}$ e angiograficamente 11 .

O vaso-espasmo dos enxertos cardíacos merece uma avaliação especial. Habitualmente, ele decorre da reatividade do enxerto a substâncias vasoconstritoras circulantes $7,12,19,20$. Esses elementos podem estar sendo administrados por via endovenosa ou decorrerem de catecolaminas endógenas. No período de pós-operatório imediato já foram detectadas substâncias com nível sérico aumentado pela circulação extracorpórea e que podem elevar o risco de vaso-espasmo, tais como o tromboxane $\mathrm{A} 2^{10,24}$ e a noradrenalina endógena 17. A reação de cada enxerto a esses elementos dependeria do número de receptores nas células de sua musculatura lisa, da capacidade de propagação do estímulo contrátil, do diâmetro do vaso sangüíneo e da espessura e composição da camada média ${ }^{23}$. Nesse último aspecto, a AR estaria em desvantagem em relação aos demais enxertos, como já citado anteriormente. Entretanto, deve-se considerar o maior diâmetro de sua luz frente às demais artérias. Diferenças no vasa-vasorum também podem levar a suscetibilidades diferentes dos vasos à isquemia. A AR tem sua vasa-vasorum localizada preferencialmente na camada adventícia e sua retirada para enxerto livre não prejudicaria diretamente a nutrição da camada média ${ }^{15}$. 0 mesmo não ocorre com A AGED, em que o vasa-vasorum penetra na camada média e cuja utilização como enxerto livre pode comprometer sua irrigação 8 .

O endotélio vascular também tem participação nos fenômenos de espasmo arterial. Em condições normais, o endotélio protege as células musculares lisas vasculares dos agentes séricos vasoconstritores, através da liberação de óxido nítrico ${ }^{16}$. Entretanto, em situações em que há aumento na produção de endotelina, como nos casos de ativação da coagulação, o tônus vascular pode estar incrementado, com o conseqüente comprometimento do fluxo sangüíneo ${ }^{8}$. Estudos comparativos in vitro de segmentos de $A R, A T I$ e AGE mostram maior reatividade do endotélio da AR 6 .

Todas essas evidências sugerem maior tendência ao espasmo da AR sobre os demais enxertos, enfatizando a necessidade do uso de drogas antiespasmódicas e inibidores plaquetários quando a AR for usada como enxerto livre. Esse novo enfoque permitiu a retomada da AR como enxerto coronário.

ACAR \& BRUNEVAL ${ }^{1}$, juntamente com CARPENTIER et alii ${ }^{4,5}$, relataram 153 pacientes operados com o uso da AR. Dentre 46 enxertos com AR estudados num período de 6 meses a 24 meses (média =10,1 meses) de pós-operatório, $41(89,1 \%)$ estavam pérvios e sem anormalidades. Três $(6,5 \%)$ estavam ocluídos, $1(2,2 \%)$ com estenose na anastomose distal (passível de angioplastia) e 1 $(2,2 \%)$ com estenose moderada.

Ainda não temos estudo cineangiográfico a médio prazo de nossos pacientes, considerando-se o recente início do procedimento. Entretanto, todos os 30 pacientes permanecem assintomáticos e os exames complementares não sugerem espasmo ou oclusão do enxerto.

O cateterismo precoce (antes da alta hospitalar) realizado em $7(23,3 \%)$ de nossos pacientes demonstrou boa perviabilidade e ausência de espasmos, inclusive no realizado em associação com a endarterectomia da artéria coronária direita.

Outro aspecto importante a ser destacado é a conseqüência, para o antebraço e para a mão, com a retirada da artéria. Os estudos anatômicos sugerem que a mão é amplamente irrigada pelos sistemas radial e ulnar, que se intercomunicam e se suprem mutuamente quando necessário. SLOGOFF et alii ${ }^{18}$ estudaram 1699 pacientes submetidos a cirurgia cardiovascular, nos quais houve catete- 
DALLAN, L. A.; OlIVEIRA, S. A.; CORSO, R. C.; PEREIRA, A. N.; IGLÉSIAS, J. C. R.; VERGINELLI, G.; JATENE, A. D. Revascularizaçāo do miocárdio com a artéria radial. Rev. Bras. Cir. Cardiovasc., 10 (2): 77-83, 1995.

rização da AR. Os exames clínico e vascular com o auxílio do Doppler, realizados de 1 a 7 dias após a retirada do cateter, demonstraram a oclusão da AR em $25 \%$ dos pacientes. Nenhum deles teve qualquer alteração isquêmica no membro, mesmo nos 16 em que o teste de Allen mostrava-se anormal, e ainda assim tiveram sua AR cateterizada no pré-operatório.

O teste de Allen é sugerido para detectar o fluxo colateral advindo da artéria ulnar não suficiente para suprir a oclusão da AR. Apesar das críticas pela elevada incidência de falsos positivos ${ }^{14}$, ele constitui manobra simples e que, juntamente com o exame Doppler, deve ser realizado no pré-operatório.

Não observamos nenhuma manifestação isquêmica no antebraço ou na mão em nossos pacientes; observamos em 1 parestesia transitória do polegar, sem déficit funcional, coincidindo com a presença de hematoma e necessidade de revisão cirúrgica local. Essas parestesias do polegar são explicadas pelo traumatismo cirúrgico do ramo superficial do nervo radial ${ }^{3}$.

Em todos os 30 pacientes da série a $A R$ foi aproveitada, e não observamos sinais macroscópicos de aterosclerose. Isso vem de encontro com o relato da literatura. Dentre 122 AR utilizadas por ACAR et alii ${ }^{3}$, em apenas 1 paciente diabético havia calcificação junto à prega do punho, o que não impediu o seu emprego, utilizando-se a porção mais proximal. É interessante observar que $20 \%$ desses pacientes tinham múltiplos locais de aterosclerose e que 15 deles já haviam sofrido cirurgias vasculares.

A AR parece constituir uma alternativa de grande importância na revascularização do miocárdio, especialmente como complemento da ATI, na busca da revascularização completa com enxertos arteriais. Outro enxerto arterial, como a AGED, também deve ser considerado Evidências de espasmos constatados angiograficamente no pós-operatório imediato, e que desapareceram em estudo após 6 a 12 meses, sugerem que o enxerto com AR pode ter seu tono vascular diminuído com o tempo ${ }^{3}$. A administração de bloqueadores dos canais de cálcio torna-se obrigatória nesse período inicial. Apenas o seguimento a longo prazo dos pacientes e a realização de maior número de estudos nos trarão subsídios para que possamos tirar conclusões definitivas.

RBCCV 44205-262

DALLAN, L. A.; OLIVEIRA, S. A.; CORSO, R. C.; PEREIRA, A. N.; IGLÉZIAS, J. C. R.; VERGINELLI, G.; JATENE, A. D. - Radial artery for coronary artery bypass grafting. Rev. Bras. Cir. Cardiovasc., 10 (2): $77-83,1995$

ABSTRACT: The radial artery (RA) was used as a conduit for coronary artery bypass many years ago. Some years later, the graft was abandoned due to of a high incidence of narrowing or occlusion. The advent of new antispastic drugs led us to reinvestigate the use of the RA for coronary artery bypass grafting. Since May 1994, 30 patients underwent myocardial revascularization using 31 RA grafts (1 patient received 2 grafts) at our Service. The left internal thoracic artery (LITA) was concomitantly used in all $(100 \%)$ patients, the right internal thoracic artery (RITA) in $9(30 \%)$ patients and a saphenous vein graft in $24(80 \%)$ cases. A mean of 3.5 graft per patient was performed. The RA was anastomosed to the diagonal $(n=10 / 33,3 \%)$, circunflex $(n=8 /$ $26.6 \%)$, right coronary $(n=8 / 26.6 \%)$, diagonalis $(n=4 / 13.3 \%)$ and anterior interventricular artery $(n=1 / 3.3 \%)$. Two $(6.6 \%)$ patients presented for redo coronary surgery and $14(46 \%)$ had prior myocardial infarction. Two patients underwent associated ventricular aneurismectomy and 3 coronary endarterectomy. The left RA was used in $28(93.4 \%)$ patients, and the right RA in the $2(6.6 \%)$ remaining. The RA was used as a free graft. The proximal end of the RA was directly anastomosed to the ascending aorta using a 7-0 Polypropylene suture. After complete, the aortic clamp was removed and the blood flow throught the RA was tested. The distal anastomosis was then performed using a running 7-0 Polypropylene suture. All patientes received diltiazem started intraoperatively and continued at the follow-up period, when the AAS was associated. There was no mortality in this series. Angiographic controls were obtained in $7(23.3 \%)$ patients before the Hospital discharge, with all patent grafts without any abnormality. We have not observed any ischemic symptoms of the hand in this series. We believe that the RA is a valuable alternative procedure to the myocardial revascularization, specially after the advent of new antispastic drugs. However, a larger study including late control angiograms is still required to establish definitive conclusions.

DESCRIPTORS: Radial artery, myocardial revascularization. Myocardial revascularization, surgery, radial artery.

ADENDO: Desde o fechamento deste trabalho, foram operados mais 64 pacientes, totalizando 94 casos. 0 resultado destes últimos casos reforçam os achados anteriores. 
DALLAN, L. A.; OliVEIRA, S. A.; CORSO, R. C.; PEREIRA, A. N.; IGLÉSIAS, J. C. R.; VERGINELLI, G.; JATENE, A. D. Revascularização do miocárdio com a artéria radial. Rev. Bras. Cir. Cardiovasc., 10 (2): 77-83, 1995.

\section{REFERÊNCIAS BIBLIOGRÁFICAS}

1 ACAR, C. \& BRUNEVAL, P. - Reply to the editor. Ann. Thorac. Surg. 55: 1597-1598, 1993.

2 ACAR, C.; JEBARA, V. A.; PORTOGHESE, M. Comparative anatomy and histology of the radial artery and the internal thoracic artery: implication for coronary bypass. Surg. Radiol. Anat., 13: 283-288, 1991.

3 ACAR, C.; JEBARA, V. A.; PORTOGHESE, M.; BEYSSEN, B.; PAGNY, J. Y.; GRARE, P.; CHACHQUES, J. C.; FABIANI, J.; DELOCHE, A.; GUERMONPREZ, J. L.; CARPENTIER, A. F. Revival of the radial artery for coronary bypass grafting. Ann. Thorac. Surg., 54: 652-660, 1992.

4 CARPENTIER, A. - Discussion of GEHA, A. S.; KRONE, R. J.; McCORMICK, J. R.; BAUE, A. E. - Selection of coronary bypass: anatomic, physiological and angiographic considerations of vein and mammary artery grafts. J. Thorac. Cardiovasc. Surg., 70: 414$431,1975$.

5 CARPENTIER, A.; GUERMONPREZ, J. L.; DELOCHE, A.; FRECHETTE, C.; DUBOST, C. - The aorta-tocoronary radial artery bypass graft: a technique avoiding pathological changes in grafts. Ann. Thorac. Surg., 16: 111-121, 1973.

6 CHARDIGNY, C.; JEBARA, V. A.; ACAR, C.; DESCOMBES, J.; VERBEUREN, T. J.; CARPENTIER, A.; FABIANI, J. N. - Vasoreactivity of the radial artery: comparison with the internal mammary and gastroepiploic arteries with implications for coronary artery surgery. Circulation, 88 (Supl. 2): 115-127, 1993.

7 CHIU, C. J. - Why do radial artery grafts for aortocoronary bypass fail?: a reappraisal. Ann. Thorac. Surg. 22: 520-523, 1976.

8 COSTELLO, K. B.; STEWART, D. J.; BAFFOUR, R. Endothelin is a potent constrictor of humain vessels used in coronary revascularizations surgery. Eur. $J$. Pharmacol., 186: 311-314, 1990.

9 CURTIS, J. J.; STONEY, W. S.; ALFORD, W. C.; BURRUS, G. R.; THOMAS, C. S. - Intimal hyperplasia: a cause of radial artery aortocoronary bypass graft failure. Ann. Thorac. Surg., 20: 628$635,1975$.

DAVIES, G. C.; SOBEL, M.; SALZMAN, E. W. - Elevated plasma fibrinopeptide $A$ and thromboxane B2 levels during cardiopulmonary bypass. Circulation, 61: 808 $814,1980$.

11 FISK, R. L.; BROOKS, C. H.; CALLAGHAN, J. C.; DVORKIN, J. - Experience with the radial artery graft for coronary bypass. Ann. Thorac. Surg., 21; 513$518,1976$.

HE, G. W.; ROSENFELDT, F. L.; BUXTON, B. F.;
ANGUS, J. A. - Reactivity of human isolated internal mammary artery to constrictor and dilator agents: implications for treatment of internal artery spasm. Circulation, 80 (Supl. 1): 142-150, 1989.

13 JEBARA, V. A.; CHARDIGNY, C.; ACAR, C. Vasoreactivity of the radial artery: comparison with the internal mammary artery and the gastroepiploic arteries: implications for coronary artery bypass. Circulation, 86 (Supl. 1): 179, 1992.

14 KAMINSKI, R. W. \& BARNES, R. W. - Critique of the Allen test for continuity of the palmar arch assessed by Doppler ultrasound. Surg. Gynecol. Obstet., 143: $861-864,1976$.

15 MASSA, G.; JOHANSON, S.; KIMBLAD, P. O.; SJOBERG, T.; STEEN, S. - Might free arterial grafts fail due to spasm? Ann. Thorac. Surg., 51: 94-101, 1991.

16 PALMER, R. M. J.; FERRIGE, A. G.; MONCADA, S. Nitric oxide release accounts for the biological activity of endotelium - derived relaxing factor. Nature, 327: 524-526, 1987.

17 REVES, J. G.; KARP, R. B.; BUTTNER, E. E.; TESONE, S.; SMITH, L. R.; SAMUELSON, P. N.; KREUSCH, G. R.; OPARIL, S. - Neuronal and adrenomedullary catecholamine release in response to cardiopulmonary bypass in man. Circulation, 66: 49-55, 1992.

18 SLOGOFF, S.; KEATS, A. S.; ARLUND, C. - On the safety of radial artery cannulation. Anesthesiology, 59: $42-47,1983$.

19 SUMA, H. - Spasm of the gastroepiploic artery graft. Ann. Thorac. Surg., 49: 168-169, 1990.

20 SURABU, M. R.; McCHUNG, J. A.; FASS, A.; REED, G. E. - Early postoperative spasm in left internal mammary artery bypass grafts. Ann. Thorac. Surg. 44: 199-200, 1987.

21 VAN SON, J. A. M. \& SMEDTS, F. - Revival of the radial artery for coronary artery bypass graft. Ann. Thorac. Surg., 55: 1596-1597, 1993. (Carta ao Editor).

22 VAN SON, J. A. M.; SMEDTS, F.; De WILDE, P. C. M. - Histological study of the internal mammary artery with emphasis on its suitability as a coronary artery bypass graft. Ann. Thorac. Surg., 55: 106-113, 1993.

23 VAN SON, J. A. M.; SMEDTS, F.; VINCENT, J. G.; VAN LIER, H. J. J.; KUBAT, K. - Comparative anatomic studies of various arterial conduits for myocardial revascularization. J. Thorac. Cardiovasc. Surg., 99: 703-707, 1990.

24 YLIKORKALA, O.; SAARELLA, E.; VIINIKKA, L. Increased prostacyclin and thromboxane production in man during cardiopulmonary bypass. J. Thorac. Cardiovasc. Surg., 82: 245-247, 1981. 